\title{
Verified nonlinear model of piezoelectric energy harvester
}

\author{
Ondrej Rubes ${ }^{1, *}$, Martin Brablc ${ }^{1}$, and Zdenek Hadas ${ }^{1}$ \\ ${ }^{1}$ Brno University of Technology, Czech Republic
}

\begin{abstract}
Energy harvesting is an important topic today. Complex monitoring systems with many nodes need energy sources and vibration energy harvesters (VEHs) could be one type of them. Mathematical model of the VEH is necessary instrument to estimate possible harvested power. This paper deals with piezoelectric VEH in setting as cantilever beam with tip mass. Traditional linear model of this type of VEH is simple, however, it represents the VEH only in one operating point and in another one (another amplitude of excitation vibrations) it could return wrong results. The nonlinear model of VEH is introduced in this paper with its parameters estimation. The nonlinear model is compared with linear model and experiment to demonstrate difference between them in amplitude frequency characteristics. Finally, the average harvested power from harmonic vibrations is measured experimentally and compared with prediction from linear and nonlinear model.
\end{abstract}

\section{Introduction}

Energy harvesting is an important topic today due to increasing numbers of IoT applications [1]. Complex monitoring systems with many nodes need energy sources and vibration energy harvesters (VEHs) could be one type of them [2]. This device uses ambient energy of vibration to harvest electric energy [3]. VEHs might be divided in two major groups [4]: piezoelectric VEHs and electromagnetic VEHs, where electromagnetic VEHs are convenient for low frequencies and piezoelectric VEHs are intended for higher frequencies and are generally smaller. This paper deals with the piezoelectric one, which is commonly composed from cantilever beam, piezoelectric layers and added tip mass [5]. Piezoelectric effect is described by matrix constitutive equations [6] that are very complex, however, it can be simplified for cantilever beam to two simple equations, which are used in this paper. This simplification reduces the model to 1 DOF oscillator with linear stiffness and linear damping [7]. In this paper will be demonstrated on experiment that this linear model is not precise enough and improved nonlinear model will be introduced. Finally, comparison of average harvested power estimated by linear and nonlinear model will be compared with experiment.

\footnotetext{
*Corresponding author: ondrej.rubes@vut.cz
} 


\section{Linear model of piezoelectric VEH}

Cantilever beam type piezoelectric VEHs are the most frequently modelled as 1 DOF linear oscillator with piezoelectric coupling [8] through equations:

$$
\begin{gathered}
m \cdot \ddot{z}+d \cdot \dot{z}+k \cdot z+\Theta \cdot v=-m \cdot \ddot{y} \\
\dot{v}=\frac{1}{c} \cdot\left(\Theta \cdot \dot{z}-\frac{v}{R}\right)
\end{gathered}
$$

Where $z$ is tip displacement (in meters), $m$ is reduced mass (in kilograms), $d$ is reduced mechanical damping (in newton seconds per meter), $k$ is reduced mechanical stiffness (in newtons per meter), $\Theta$ is piezoelectric coupling coefficient (in newtons per volt), $\ddot{y}$ is excitation acceleration (in meters per second power two), $C$ is capacitance of piezoelectric layers (in farads) and $R$ is resistivity of electric load (in ohms).

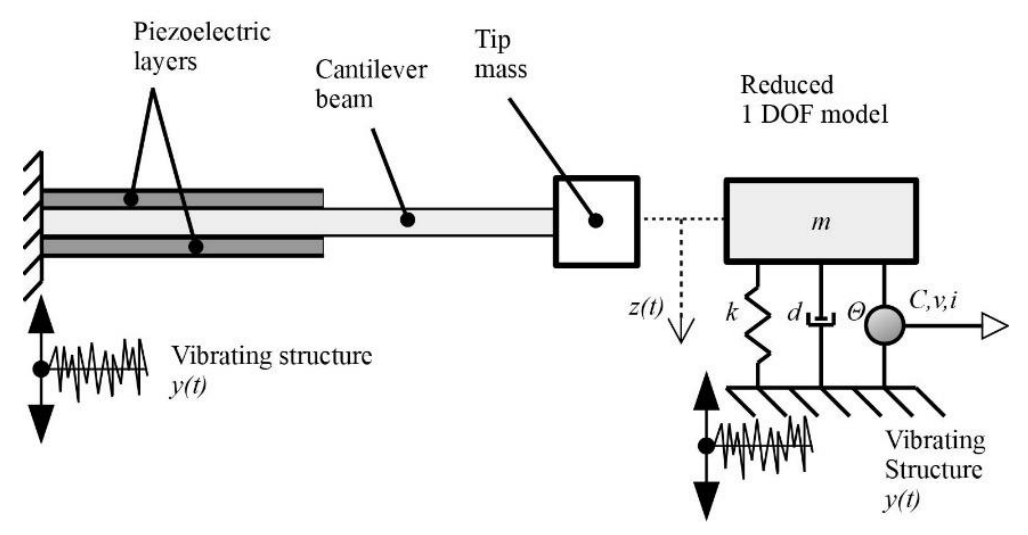

Figure 1. Piezoelectric VEH and 1 DOF model

Figure 1 illustrates schema of cantilever beam type piezoelectric VEH with its 1 DOF model. The piezoelectric VEH Mide V21B1 was used for model evaluating in this paper; Table 1 includes parameters of this model.

Table 1. Linear model parameters.

\begin{tabular}{|l|c|}
\hline Parameter & Value \\
\hline$m$ - mass & $5.2 \mathrm{~g}$ \\
\hline$d$ - mechanical damping coefficient & $0.022 \mathrm{Nsm}^{-1}$ \\
\hline$k$-stiffness & $235 \mathrm{Nm}^{-1}$ \\
\hline
\end{tabular}

\subsection{Short circuit testing}

Short circuit testing is optimal for mechanical parameter estimation because the voltage is zero, so the equation (2) can be omitted. Figure 2 shows comparison of simulated and experimentally measured amplitude-frequency (AF) characteristic for different excitation frequencies. Linear model could be tuned only for one amplitude of excitation vibrations. AF characteristic for higher amplitude of excitation demonstrates that linear model is not precise enough, because there is difference between model and experiment in resonance frequency $(2 \%)$ and in amplitude of resonance (30\%). The nonlinear model must be used to improve it. 


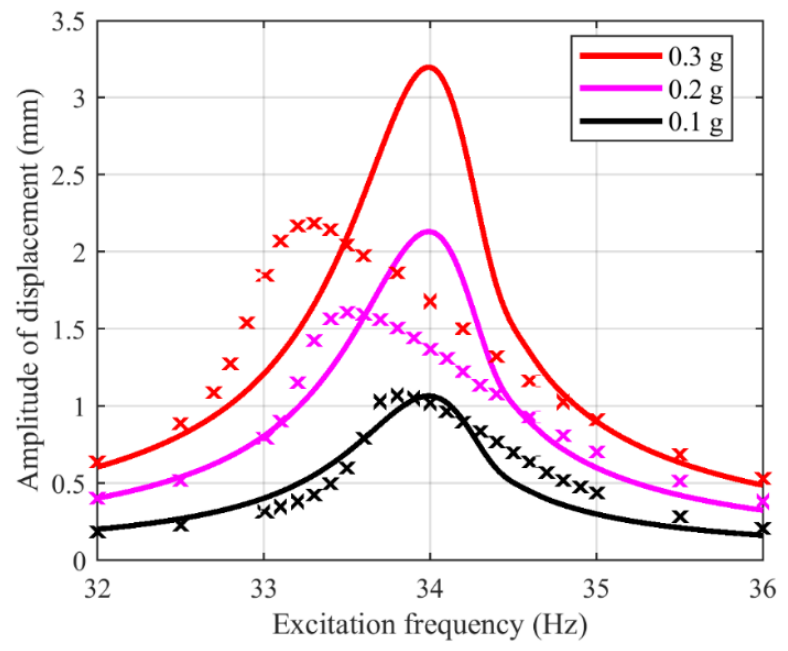

Figure 2. AF characteristics obtained from simulations (solid lines) and from experiments (points) for different amplitudes of excitation vibrations

\section{Nonlinear model with cubic stiffness and cubic damping}

Linear model might be extended to nonlinear through nonlinear damping and nonlinear stiffness. Simple way is to extend equation with cubic damping and cubic stiffness:

$$
m \cdot \ddot{z}+d \cdot \dot{z}+d_{3} \cdot \dot{z}^{3}+k \cdot z+k_{3} \cdot z^{3}+\Theta \cdot v=-m \cdot \ddot{y}
$$

Where $d_{3}$ is cubic damping coefficient $\left(\mathrm{Ns}^{3} \mathrm{~m}^{-3}\right)$ and $k_{3}$ is cubic stiffness coefficient $\left(\mathrm{Nm}^{-3}\right)$. Reduced mass $m$ is known and parameters $d, d_{3}, k, k_{3}$ must be estimated.

\subsection{Parameter estimation}

The nonlinear model has two novel parameters: $d_{3}$ and $k_{3}$, which must be estimated [9]. The original linear parameters $d$ and $k$ must be re-estimated, however, the linear model values can be used as the first approximation. Parameter estimation in this case is problem of searching the state space of four parameters with initial approximation of linear parameters and knowledge that nonlinear stiffness parameter should be negative (experimental characteristic is softening) and nonlinear damping coefficient should be positive, because the higher amplitude in experiment is more damped. Final estimated parameters are in table 2.

Table 2. Nonlinear model parameters.

\begin{tabular}{|l|c|}
\hline Parameter & Value \\
\hline$m$ - mass & $5.2 \mathrm{~g}$ \\
\hline$d$ - mechanical damping coefficient & $0.017 \mathrm{Nsm}^{-1}$ \\
\hline$d_{3}$ - nonlinear mechanical damping coefficient & $0.12 \mathrm{Ns}^{3} \mathrm{~m}^{-3}$ \\
\hline$k$ - stiffness & $238 \mathrm{Nm}^{-1}$ \\
\hline$k_{3}-$ cubic stiffness coefficient & $-3 \cdot 10^{6} \mathrm{Nm}^{-3}$ \\
\hline
\end{tabular}

Figure 3 demonstrates correlation between experiment and nonlinear model. It is obvious that nonlinear model is more precise that linear one (compare with figure 2). There is still small 
difference in AF characteristic for each amplitude of excitation, however, it is less than for linear model. The difference is less than $1 \%$.

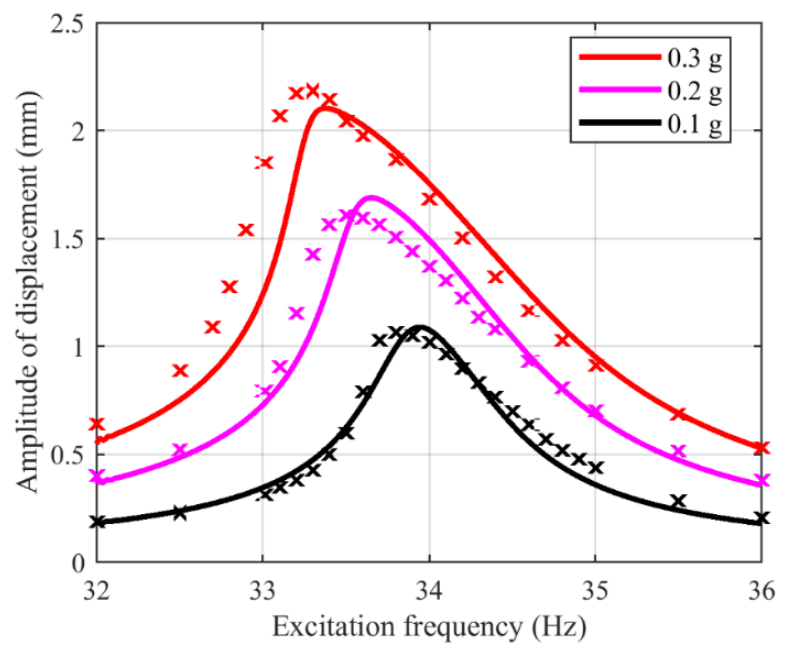

Figure 3. AF characteristics obtained from simulations (solid lines) and from experiments (points) for different amplitudes of excitation vibrations with nonlinear model

\subsection{Testing with electric load}

Piezoelectric effect is described by equation (2), which has two parameters. Electric capacity could be simply measured by capacity meter and its value is $18 \mathrm{nF}$. The piezoelectric coupling coefficient was estimates as $0.33 \mathrm{mNV}^{-1}$. Figure 4 evaluate nonlinear model with experiment. Three curves represent different resistivity of electric load with the same excitation vibrations. It might be said that model sufficiently represents real harvester.

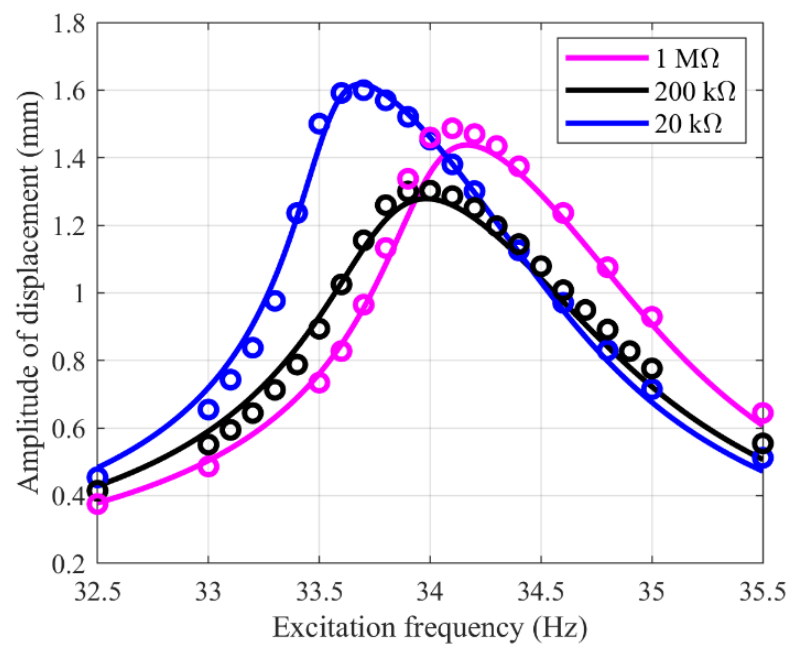

Figure 4. AF characteristics obtained from simulations (solid lines) and from experiments (points) for different electric load resistivity. 
Finally, Figure 5 demonstrates output voltage in time domain for excitation acceleration amplitude $0,2 \mathrm{~g}$ and resonance frequency. It depicts that voltage from linear model is higher than in experiment by about $10 \%$. Average harvested power in Table 3. illustrates difference between model and reality. Estimated power from linear model is $19 \%$ higher than from experiment and from nonlinear model is $4 \%$ lower. It demonstrates that nonlinear model is more precise for estimating the average output power than linear model.

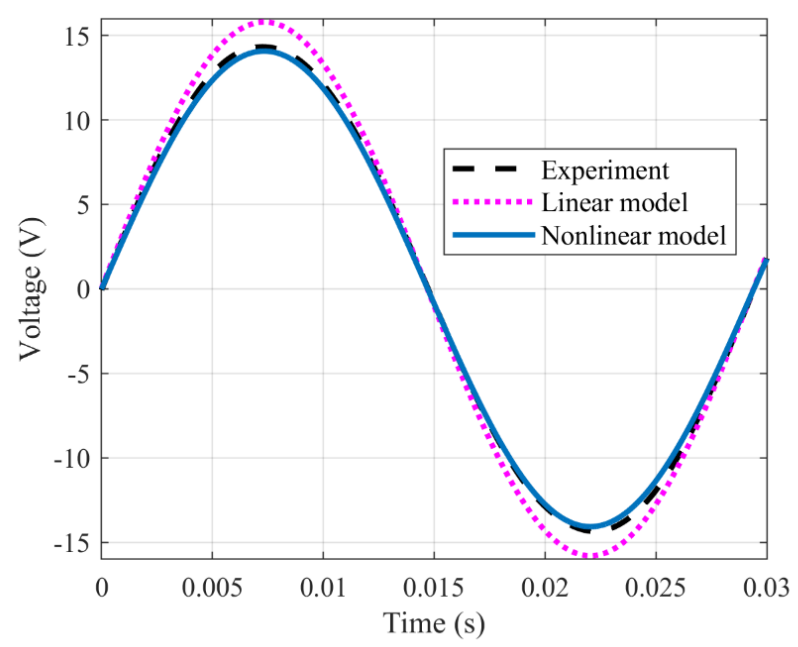

Figure 5. Voltage from linear and nonlinear model in comparison to experimental data.

Table 3. Average harvested power.

\begin{tabular}{|c|c|}
\hline Case & Average power \\
\hline Experiment & $52 \mu \mathrm{W}$ \\
\hline Linear model & $62 \mu \mathrm{W}$ \\
\hline Nonlinear model & $50 \mu \mathrm{W}$ \\
\hline
\end{tabular}

\section{Conclusion}

Energy harvesting, as important topic for complex smart systems, needs accurate models to predict possibility of harvested power. This paper demonstrated a piezoelectric VEH and its models. The first model is linear model with 1 DOF. Comparison of simulation and experiment disclosed huge difference between linear model and experiment in AF characteristic. The nonlinear model was introduced as possible improvement. This model has cubic stiffness and cubic damping. Verification demonstrated good correlation between nonlinear model and experiment in AF characteristic.

Next part of the paper dealt with output power. The nonlinear model with model of piezoelectric effect provided precise AF characteristic of whole piezoelectric VEH with various resistive loads. Finally, comparison of average harvested power from experiment, linear and nonlinear model was presented. It showed that the nonlinear model has error $4 \%$ in average output power and the linear model has error $19 \%$. What is more, the linear model predicted higher power than experiment, while the nonlinear predicted lower power. In summary, it may be said that nonlinear model of piezoelectric cantilever beam VEH is not precise enough. 
The research leading to these results has received funding from the MEYS under the National Sustainability Programme I (Project LO1202) and additionally this paper has been supported by the project "Novel material architectures for SMART piezoceramic electromechanical converters" GACR 17-08153S under Czech Science Foundation.

\section{References}

[1] C. Cepnik, R. Lausecker, and U. Wallrabe, Micromachines 4, 168 (2013).

[2] J. Chen and Z. L. Wang, Joule 1, 480 (2017).

[3] A. Khaligh, IEEE Trans. Ind. Electron. 57, 850 (2010).

[4] Z. Hadas, J. Smilek, and O. Rubes, in Proc. Vol. 10246, Smart Sensors, Actuators, MEMS VIII, edited by L. Fonseca, M. Prunnila, and E. Peiner (2017), p. 1024619.

[5] L. T.-E. Nyamayoka, G. A. Adewumi, and F. L. Inambao, J. Energy South. Africa 28, (2017).

[6] A. K. Batra and A. Alomari, Power Harvesting via Smart Materials (SPIE PRESS, 2017).

[7] Z. Hadas and R. Lan, in (2016), pp. 305-310.

[8] Y. C. Shu and I. C. Lien, Smart Mater. Struct. 15, 1499 (2006).

[9] Z. Hadas, V. Singule, and C. Ondrusek, Solid State Phenom. 164, 291 (2010). 